\title{
Elasto-plastic shape optimization using the level set method
}

\author{
Aymeric Maury $^{1}$, Grégoire Allaire ${ }^{2}$, and François Jouve ${ }^{3}$ \\ ${ }^{1}$ GIREF, Département de mathématiques et de statistique, Université Laval, Québec \\ G1V 0A6, Canada \\ ${ }^{2}$ CMAP, Ecole Polytechnique, CNRS, Université Paris-Saclay, 91128 Palaiseau, France \\ ${ }^{3}$ Laboratoire J.L. Lions (UMR CNRS 7598), University Paris Diderot, Paris, France
}

\begin{abstract}
This article is concerned with shape optimization of structures made of a material obeying Hencky's laws of plasticity, with the stress bound expressed by the von Mises effective stress. The ill-posedness of the model is circumvented by using two regularized versions of the mechanical problem. The first one is the classical Perzyna formulation which is regularized, the second one is a new regularized formulation proposed for the von Mises criterion. Shape gradients are calculated thanks to the adjoint method. The optimal shape is numerically computed by using the level set method. To illustrate the validity of the method, $2 \mathrm{D}$ examples are performed.
\end{abstract}

\section{Introduction}

As shape optimization becomes more and more popular in industrial applications, the ability to take into account nonlinear mechanical behaviours is of great need. Plasticity ones are some of the most used since the associated models account for irreversible microscopic mechanical defects. These defects result in plastic areas which tend to deform more than elastic ones. This could lead to structural integrity dangers and sometimes to breaking. In this case, the designer often tries to avoid the creation of plastic regions by controlling the internal constraints. But plasticity could also be useful when, for instance, the breaking of a piece is meant to protect other parts which are difficult to repair or which should absolutely not collapse (like the use of a circuit breaker in electricity). Eventually there are materials, called ductile, which can suffer big deformations after the elastic phase (without collapsing) and the designer can take advantage of this property by allowing plastic areas and trying to distribute the constraint in the structure the most uniformly as possible.

From a mechanical point of view, plasticity was first studied by Tresca, Saint Venant, Lévy and Bauschinger in the nineteenth century and in the next century by Prandtl, von Mises and Reuss. From a mathematical point of view the study was started by Prager, Drucker and Hill and using the theory of variational inequalities and convex analysis by Moreau [45], Duvaut and Lions [14]. Since then, a lot of articles were published on the subject investigating the well posedness of these problems. We particularly mention Suquet [64], Temam [67] and more recently Dal Maso [8], [9]. Concerning the regularity of solutions the reader is refered to [16] and [4].

This article focuses on shape optimization with the level set method for static perfect plasticity, also called the Hencky model, for the von Mises criterion (also called Huber-Mises or Huber-Mises-Hencky criterion). As pointed in [64], the Hencky plasticity is not mechanically relevant but for some very specific cases. It does not account for the path dependency shown by the experiments and thus is rather a non quadratic law. However it raises a great amount of the mathematical difficulties of the quasi-static case. Moreover, when the numerical solution of the quasi-static evolution comes into question, a time discretization leads to a sequence of Hencky model to be solved. Finally for the shape optimization it will be easier to first study this steady problem as time-dependent problems lead to backward adjoint problems.

The problem can be put under a mixed form including a variational inequation and a variational equation. Due to the appearing of a variational inequation there is no chance for this problem to 
be Fréchet or Gateaux differentiable. The solution we choose, which was largely investigated in the framework of control theory for problems with hardening but not in the framework of shape optimization, is the use of a regularized penalized problem to get rid of the variational inequality. On this issue we mention, for the static case, [24], [26], [27], [11] (using a primal formulation), [28], [5] (for a second order optimality condition), for the quasi-static case [69] and for other plastic models [10] and [36].

In shape optimization, the first case which was considered was the one of beam structure and frame optimization, which is addressed for instance in [33], [17], [66], [32], [1], [50], [35]. We point out the particular case of [74], where periodical microstructures are used. From a theoretical point of view, in [62], chapter 4.8, the shape derivative of an elasto-plastic torsion problem is computed and, in chapter 4.9 , the shape derivative of the stress tensor is calculated in the case of the visco-plastic model of Perzyna (see section 3). There also exist numerous articles in which, for a particular optimization problem, the existence of a solution is proved for the continuous and discrete case, assuming a uniform Lipschitz boundary. The discrete solution is then proved to converge to the continuous one. In [29] or in [21] and [22], the analysis is done in the framework of the Hencky model for a criterion depending only on $\sigma$. In [31], the analysis is done for axisymmetric bodies. In [30], the same analysis is done in the case of the Prandtl-Reuss model of elasto-plasticity (dynamic plasticity). In [52] strain-hardening is added. Finally [13] and [20] (for numerical results) deal with a particular elasto-plasticity model (introduced by Washizu in [71]).

From a numerical point of view some authors use conical derivatives inside a bundle algorithm to optimize the shape, see [54] and [39]. Another way to proceed is to differentiate the radial return algorithm (the generalized Newton method, see [56] chapter 8). The differentiation is analyzed in [68], [40] and [44]. This procedure was applied in various articles, using the von Mises criterion. In [43] and [57], the authors consider linear isotropic hardening/softening. They use, first, the SIMP method and finish the shape optimization using splines to parameterize the boundary and recover smooth shapes. In [37], rate-independent elasto-plasticity and contact friction are taken into account. For the shape optimization, splines are used. In [38], the same is done but for finite deformation elasto-plasticity. In [34], the elato-plastic model is the same but the derivative is computed differently. It also performs two-phase optimization, by using the SIMP method. For perfect plasticity we mention [15], using the boundary perturbation method. We also mention that based on the two regularized plasticity models presented here, we manage to extend our approach to quasi-static perfectly plastic problems.

Section 2 describes perfect plasticity from both a mechanical and a mathematical point of view, introducing the formulations and spaces classically used to study this particular behaviour. Section 3 focuses on two ways to regularize the Hencky model. The first one is the well-known Perzyna formulation and the second one is a new derived regularization adapted to the von Mises criterion. For each of them we give theorems proving existence and uniqueness of solutions as well as the convergence of the regularized solutions to the solution of static perfect plasticity. In section 4, a general shape optimization is introduced and shape gradients are computed by virtue of the adjoint method. Section 5 recalls the basis of the level set method and briefly describes how the plasticity problems are numerically solved. Finally our numerical results performed with the Scilab free software [58] are gathered in section 6 .

\section{Elasticity and perfect plasticity}

\subsection{Mechanical model}

In this paper $\Omega$ denotes an open bounded subset of $\mathbb{R}^{d}$ where $d=2$ or 3 and represents the shape of the structure we want to optimize. Its boundary is divided into three disjoint parts meaning that:

$$
\partial \Omega=\Gamma_{0} \cup \Gamma_{N} \cup \Gamma
$$

On $\Gamma_{0}$, the structure is clamped and on $\Gamma_{N}$ a force is applied. The free part of the boundary is $\Gamma$. The structure $\Omega$ is filled with a linear isotropic perfect plastic material with Hooke's law defined by a fourth-order tensor $A$ such that, for any symmetric matrix $\tau$ :

$$
A \tau=2 \mu \tau+\lambda \operatorname{Tr}(\tau) I_{d}
$$

where $\mu$ and $\lambda$ are the Lamé moduli. 


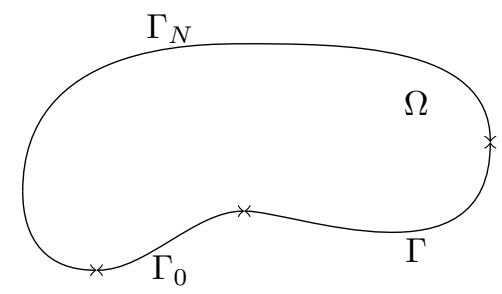

Figure 1: The open set $\Omega$.

We consider that the material follows the Hencky law [14] of plasticity which is a finer description of the material behaviour than linearized elasticity from which it is derived. We recall that in linearized elasticity the stress tensor $\sigma$ is linearly related to the strain $e(u)=\frac{1}{2}\left(\nabla u+{ }^{t} \nabla u\right)$ by $\sigma=A e(u)$. Plasticity is based on a decomposition of the strain tensor into two parts ([19], chapter 3). The first one is the elastic strain, denoted by $e_{e}$, and the second one is the plastic strain, $e_{p}$. Then we have:

$$
e(u)=e_{e}+e_{p}
$$

It has to be mentioned that if $e(u)$ is the symmetric part of the gradient of the displacement $u$, it is not the case for $e_{e}$ and $e_{p}$ which are however symmetric. The stress tensor $\sigma$ is only related to the elastic part:

$$
\sigma=A e_{e}
$$

and replacing it in (2) yields:

$$
e(u)=A^{-1} \sigma+e_{p}
$$

The other fundamental ingredient is the elastic region and the yield surface. When $\sigma$ is in a certain set called the elastic region, the plastic strain is equal to zero, $e_{p}=0$. When $\sigma$ is on the yield surface which is the boundary of the elastic region, the plastic strain can vary. In this article these regions are defined by a continuous function $\mathcal{F}$, called the yield function. Thus we define $K_{M}$ as a subset of symmetric second-order tensors:

$$
K_{M}=\left\{\tau \in \mathbb{M}_{s}^{d} \text { such that } \mathcal{F}(\tau) \leq 0\right\}
$$

with $\mathbb{M}_{s}^{d}$ the space of symmetric second-order tensors in dimension $d$. The elastic region corresponds to $\mathcal{F}(\tau)<0$ and the yield surface is defined by $\mathcal{F}(\tau)=0$. When the system is on the yield surface, we define the plastic strain $e_{p}$ by assuming the maximal plastic work (or Hill) principle:

$$
\sigma: e_{p} \geq \tau: e_{p} \quad \forall \tau \in K_{M}
$$

which implies that $e_{p}$ belongs to the normal cone of $K_{M}$. This defines the flow rule of our model. The flow rule in plasticity defines the evolution of the plastic strain $e_{p}$ with respect to $\sigma$. When the plastic strain rate is in the normal cone of $K_{M}$ (as it is the case when the maximal plastic work principle is assumed), one talks about associated plasticity. If it is not the case, one talks about non-associated plasticity. We can sum up the different equations which characterize the evolution of the perfect elastoplastic material, supposing the Hill principle satisfied:

$$
\left\{\begin{array}{llll}
e(u) & =e_{p}+A^{-1} \sigma & \\
e_{p}:(\tau-\sigma) & \leq 0 & & \\
\sigma \in K_{M} & & \\
-\operatorname{div}(\sigma) & =f & & \text { in } \Omega \\
u & =0 & & \text { on } \Gamma_{0} \\
\sigma n & =g & & \text { on } \Gamma_{N} \\
\sigma n & =0 & & \text { on } \Gamma .
\end{array}\right.
$$

The three first lines of (6) are pointwise relations in $\Omega$. On Figure 2(a), we plot, for a $1 \mathrm{D}$ case, the Hencky law and, on Figure 2(b), the corresponding quasi-static law which can be retrieved in the same 


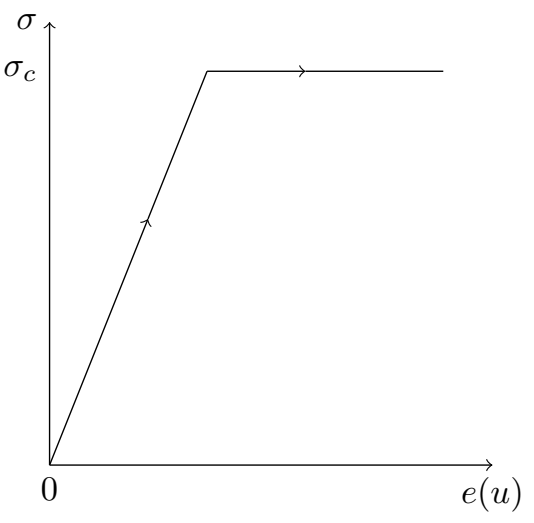

(a) Hencky's law

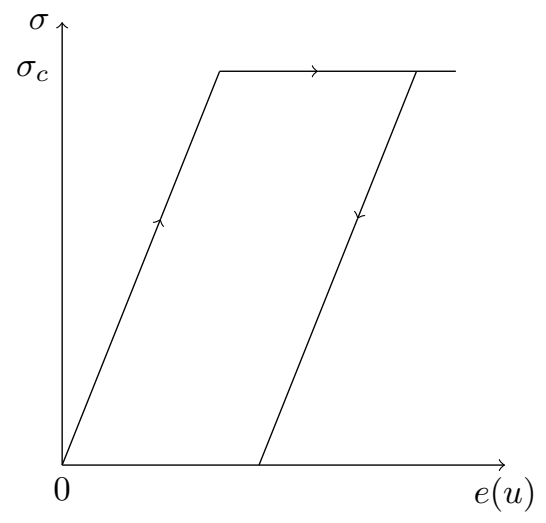

(b) Quasi-static law

way than for the static case. On a $1 \mathrm{D}$ example, the elastic region is an interval $\left[-\sigma_{c}, \sigma_{c}\right]$. When the stress $\sigma$ reaches the threshold value $\sigma_{c}$, the plastic strain, and consequently the whole strain, can increase without any growth of the stress.

\subsection{Mathematical model}

We first need to introduce some functional spaces:

$$
H_{s}(\operatorname{div}, \Omega)=\left\{\tau \in L^{2}\left(\Omega ; \mathbb{M}_{s}^{d}\right) \mid \operatorname{div} \tau \in L^{d}(\Omega)^{d}\right\}
$$

where $\mathbb{M}_{s}^{d}$ is the space of symmetric second-order tensors in dimension $d$. This space is studied in [18] or [6]. The trace operator is not defined but a normal trace operator $\gamma_{N}$ can be defined as follows: to each $\tau \in H_{s}(\operatorname{div}, \Omega)$ it associates its normal trace $\tau n \in H^{-\frac{1}{2}}\left(\partial \Omega ; \mathbb{R}^{d}\right)$. Then, for a given $g \in H^{-\frac{1}{2}}\left(\partial \Omega ; \mathbb{R}^{d}\right)$, let

$$
\Sigma_{\operatorname{div}}(g)=\left\{\tau \in H_{s}(\operatorname{div}, \Omega) \mid \gamma_{N}(\tau)=g\right\} .
$$

The space of statically admissible stresses is defined by

$$
S(f, g)=\left\{\tau \in \Sigma_{\operatorname{div}}(g) \mid-\operatorname{div} \tau=f \text { in } \Omega\right\} .
$$

Finally, introduce the convex set

$$
K=\left\{\tau \in L^{2}\left(\Omega ; \mathbb{M}_{s}^{d}\right) \mid \tau(x) \in K_{M} \text { a.e in } \Omega\right\},
$$

where $K_{M}$ is defined by (5), and the space of displacements

$$
H_{\Gamma_{0}}^{1}(\Omega)^{d}=\left\{u \in H^{1}(\Omega)^{d} \text { such that } u=0 \text { on } \Gamma_{0}\right\} .
$$

Assume $\sigma \in S(f, g)$ and, at first, $u \in H_{\Gamma_{0}}^{1}(\Omega)^{d}$. In the sequel we give two different formulations of problem (6).

\subsubsection{The dual problem}

The first formulation is independent of $u$. It was established in [67] and produces a minimization problem solved by $\sigma$ :

$$
\sigma=\operatorname{argmin}_{\tau \in S(f, g)} \psi^{*}(\tau)
$$

with $\psi^{*}(\sigma)=\frac{1}{2} A^{-1} \sigma: \sigma+\mathbb{1}_{K_{M}}(\sigma)$ and $\mathbb{1}_{K_{M}}$ the indicator function of $K_{M}$. The dual problem $(7)$ can also be written under the form

$$
\left\{\begin{array}{l}
\max _{\sigma \in K}-\frac{1}{2} \int_{\Omega} A^{-1} \sigma: \sigma d x \\
\text { under the constraint } \\
\int_{\Omega} \sigma: \epsilon(v) d x=\int_{\Omega} f \cdot v d x+\int_{\Gamma_{N}} g \cdot v d s, \forall v \in H_{\Gamma_{0}}^{1}(\Omega)^{d}
\end{array}\right.
$$


which admits a variational inequality formulation: find $\sigma \in S(f, g) \cap K$ such that for every $\tau \in S(f, g) \cap K$,

$$
\int_{\Omega} A^{-1} \sigma:(\tau-\sigma) d x \geq 0
$$

In [14] section 6.3 chapter 5 , the computations which enable to pass from (6) to (9) (and consequently to (8)) are given (see also [67] chapter 1 section 3.2). Theorem 4.1 in [67] or theorem 6.1 in [14] give the existence and uniqueness of a solution.

Theorem 2.2.1. If $S(f, g) \cap K \neq \emptyset$, problem (8) has a unique solution

\subsubsection{The displacement problem}

At first glance, problem (6) can also be written under the following variational formulation coupling a variational inequality with a variational equation:

$$
\left\{\begin{array}{l}
\int_{\Omega} \sigma: e(v) d x=\int_{\Omega} f \cdot v d x+\int_{\Gamma_{N}} g \cdot v d s, \forall v \in H_{\Gamma_{0}}^{1}(\Omega)^{d} \\
\int_{\Omega} A^{-1} \sigma:(\tau-\sigma) d x \geq \int_{\Omega} e(u):(\tau-\sigma) d x, \forall \tau \in K .
\end{array}\right.
$$

Since the variational inequality in (10) is the characterization of the projection on $K$ with the norm associated with the scalar product of $A^{-1}$, denoting by $P_{K}^{A^{-1}}$ this projection, it implies that

$$
\sigma=P_{K}^{A^{-1}}(A e(u))
$$

and (10) is equivalent to

$$
\int_{\Omega} P_{K}^{A^{-1}}(A e(u)): e(v) d x=\int_{\Omega} f \cdot v d x+\int_{\Gamma_{N}} g \cdot v d s, \quad \forall v \in H_{\Gamma_{0}}^{1}(\Omega)^{d} .
$$

Unfortunately, it is not possible to prove the existence of a solution to (12) in the space $H_{\Gamma_{0}}^{1}(\Omega)^{d}$. On this subject, we refer to [56] chapter 23.6 or [72] for a mathematical explanation. Another way to understand this issue is to come back to the mechanical meaning of plasticity and the fact that it models dislocations in the material which can produce displacement discontinuities through $(d-1)$-dimensional surfaces (see [64], chapter 6 in [67], chapter $V$ section 3.4 in [46]). To circumvent this difficulty a space of possibly discontinuous displacements was introduced in [63] and [67]: it is the space of bounded deformation $B D(\Omega)$, which is similar to the space of bounded variation $B V(\Omega)$, and is defined by

$$
B D(\Omega)=\left\{u \in L^{1}(\Omega)^{d} \mid e(u) \in M_{1}\left(\Omega ; \mathbb{M}_{s}^{d}\right)\right\}
$$

with $M_{1}\left(\Omega ; \mathbb{M}_{s}^{d}\right)$ being the space of bounded measures on $\Omega$, with values in the space of $d \times d$ symmetric tensors. The correct formulation is then: find $(\sigma, u) \in K \times B D(\Omega)$ such that:

$$
\left\{\begin{array}{l}
\int_{\Omega} \sigma: e(v) d x=\int_{\Omega} f \cdot v d x+\int_{\Gamma_{N}} g \cdot v d s, \forall v \in H_{\Gamma_{0}}^{1}(\Omega)^{d} \\
\int_{\Omega} A^{-1} \sigma:(\tau-\sigma) d x \geq{ }_{L^{\frac{d}{d-1}}}\langle u, \operatorname{div}(\tau-\sigma)\rangle_{L^{d}}, \quad \forall \tau \in K \cap \Sigma_{\operatorname{div}}(g) .
\end{array}\right.
$$

The brackets $\frac{d}{L^{\frac{d}{d-1}}}\langle,\rangle_{L^{d}}$ denote the duality product between $L^{\frac{d}{d-1}}(\Omega)^{d}$ and $L^{d}(\Omega)^{d}$. We refer to [64] and [41] for a study of this formulation, especially [64] for the definition of an external trace and the meaning of Dirichlet boundary conditions. We only recall the following existence theorem.

Theorem 2.2.2. Let $\Omega$ be a smooth domain of class $C^{2}, f \in L^{\infty}(\Omega)^{d}, g \in L^{\infty}\left(\Gamma_{N}\right)^{d}$. If $\Gamma_{0}$ is not empty and the following safe-load condition is fulfilled:

$$
\exists \bar{\sigma} \in S(f, g), \epsilon>0 \text { such that } \forall \xi \in \mathbb{M}_{s}^{d} \text { with }|\xi| \leq \epsilon, \bar{\sigma}(x)+\xi \in K_{M} \text { a.e. in } \Omega
$$

then the displacement problem (13) has a solution in $u \in B D(\Omega)$.

Remark 2.2.1. Note that there is no uniqueness of the displacement and that a counter-example can be found in [64]. 


\section{Regularizations}

As far as shape optimization is concerned, it is crucial to work on well-posed problems, which is not the case in perfect plasticity for the displacement $u$. This is the reason why a large part of the literature on shape optimization focuses on the formulation (8). Moreover, if we want to use gradient type algorithm, the solutions need to be differentiable with respect to the shape. This is clearly not the case in our problem, at least because of the projection formulation. So we have to cope with two different problems: the first one is the fact that the problem does not have a unique solution, the second one comes from the fact that the problem is not differentiable with respect to the shape.

We propose two ways to address these two difficulties. These two ways involve the projection $P_{K}^{A^{-1}}$ which has to be regularized. As an analytic expression is known for the particular case of the von Mises criterion, we restrain ourselves to this criterion. From now on the function $\mathcal{F}$ is:

$$
\mathcal{F}(\sigma)=\sqrt{\sigma_{D}: \sigma_{D}}-\sigma_{c}=|\sigma|_{D}-\sigma_{c}
$$

where, for a symmetric tensor $\sigma$, its deviatoric part $\sigma_{D}$ and hydrostatic part $\sigma_{H}$ are defined by

$$
\sigma_{D}=\sigma-\frac{\operatorname{Tr}(\sigma)}{d} I, \quad \sigma_{H}=\frac{\operatorname{Tr}(\sigma)}{d} I .
$$

Then the projection is:

$$
P_{K}^{A^{-1}}(\tau)=\tau-\max \left(0,1-\frac{\sigma_{c}}{\left|\tau_{D}\right|}\right) \tau_{D}
$$

It is now easy to regularize this projection by introducing a regularization of the function $x \rightarrow \max (0, x)$ which we note $f_{\gamma}, \gamma>0$ being a regularization parameter. Here, we choose:

$$
f_{\gamma}(x)= \begin{cases}\frac{1}{4 \gamma} x^{2}+\frac{1}{2} x+\frac{\gamma}{4} & x \in[-\gamma, \gamma] \\ \max (x, 0) & \text { otherwise }\end{cases}
$$

and the regularized projection is:

$$
P_{K}^{\gamma}(\tau)=\tau-f_{\gamma}\left(1-\frac{\sigma_{c}}{\left|\tau_{D}\right|}\right) \tau_{D}
$$

For the sake of simplicity, the pointwise convex set $K_{M}$, defined by (5), is denoted by $K$ in the sequel when no confusion can arise.

\subsection{Perzyna penalization}

\subsubsection{Formulation of the problem}

To introduce the Perzyna penalization, we use the Moreau-Yosida approximation [56] of the indicator function of $K$ in $(7), \mathbb{1}_{K}^{\eta}$ :

$$
\mathbb{1}_{K}^{\eta}(\sigma)=\frac{1}{2 \eta} \int_{\Omega} A^{-1}\left(\sigma-P_{K}^{A^{-1}}(\sigma)\right):\left(\sigma-P_{K}^{A^{-1}}(\sigma)\right) d x=\frac{1}{2 \eta}\left\|\sigma-P_{K}^{A^{-1}}(\sigma)\right\|_{A^{-1}}^{2} .
$$

From (19) we can deduce an approximation for the condition $e_{p} \in \partial \mathbb{1}_{K}(\sigma)$. As $\sigma \rightarrow \mathbb{1}_{K}^{\eta}(\sigma)$ is Fréchet differentiable (theorem 4.1 in [75]), its subdifferential reduces to its gradient and we can write the following pointwise formula for the approximation $e_{p}^{\eta}$ :

$$
e_{p}^{\eta}=\frac{1}{\eta} A^{-1}\left(\sigma-P_{K}^{A^{-1}}(\sigma)\right)
$$

This directly leads to the following system of variational equations by integration of the plasticity equations (6), replacing the condition on $e_{p}$ by (20):

$$
\left\{\begin{array}{l}
\int_{\Omega} \sigma^{\eta}: e(v) d x=\int_{\Omega} f \cdot v d x+\int_{\Gamma_{N}} g \cdot v d s, \forall v \in H_{\Gamma_{0}}^{1}(\Omega)^{d} \\
\int_{\Omega} A^{-1} \sigma^{\eta}: \tau d x+\frac{1}{\eta} \int_{\Omega} A^{-1}\left(\sigma^{\eta}-P_{K}^{A^{-1}}\left(\sigma^{\eta}\right)\right): \tau d x=\int_{\Omega} e\left(u^{\eta}\right): \tau d x, \quad \forall \tau \in L^{2}\left(\Omega ; \mathbb{M}_{s}^{d}\right) .
\end{array}\right.
$$


This also gives rise to further simplification of the problem (21) which is a mixed variational problem. Indeed, as we shall now show, the variable $\sigma$ can be eliminated. From (20) we can deduce the expression of $\sigma^{\eta}$ :

$$
\sigma^{\eta}=A e\left(u^{\eta}\right)-\frac{1}{\eta}\left(\sigma^{\eta}-P_{K}^{A^{-1}}\left(\sigma^{\eta}\right)\right) .
$$

This expression says that $A e\left(u^{\eta}\right)$ is on the ray defined by $\sigma^{\eta}$ and its projection. So it implies that (chapter 3 lemma $3.2[56])$ :

$$
P_{K}^{A^{-1}}\left(\sigma^{\eta}\right)=P_{K}^{A^{-1}}\left(A e\left(u^{\eta}\right)\right) .
$$

This enables to write $\sigma^{\eta}$ with respect to only $u^{\eta}$ and transform the implicit definition into an explicit one:

$$
\sigma^{\eta}=\frac{\eta}{1+\eta} A e\left(u^{\eta}\right)+\frac{1}{1+\eta} P_{K}^{A^{-1}}\left(A e\left(u^{\eta}\right)\right) .
$$

We can write the new nonlinear variational equation of the Perzyna visco-elastoplasticity:

$$
\int_{\Omega}\left(\frac{\eta}{1+\eta} A e\left(u^{\eta}\right)+\frac{1}{1+\eta} P_{K}^{A^{-1}}\left(A e\left(u^{\eta}\right)\right)\right): e(v) d x=\int_{\Omega} f \cdot v d x+\int_{\Gamma_{N}} g \cdot v d s \forall v \in H_{\Gamma_{0}}^{1}(\Omega)^{d} .
$$

It remains to regularize the projection $P_{K}^{A^{-1}}$, replacing it by $P_{K}^{\gamma}$ defined by (18): find $u_{\eta}^{\gamma} \in H_{\Gamma_{0}}^{1}(\Omega)^{d}$ such that,

$$
\int_{\Omega}\left(\frac{\eta}{1+\eta} A e\left(u_{\eta}^{\gamma}\right)+\frac{1}{1+\eta} P_{K}^{\gamma}\left(A e\left(u_{\eta}^{\gamma}\right)\right)\right): e(v) d x=\int_{\Omega} f \cdot v d x+\int_{\Gamma_{N}} g \cdot v d s \forall v \in H_{\Gamma_{0}}^{1}(\Omega)^{d} .
$$

or in a mixed form: find $\sigma_{\gamma}^{\eta} \in L_{s}^{2}(\Omega)^{d \times d}$ and $u_{\gamma}^{\eta} \in H_{\Gamma_{0}}^{1}(\Omega)^{d}$ such that:

$$
\left\{\begin{array}{l}
\int_{\Omega} \sigma_{\gamma}^{\eta}: e(v) d x=\int_{\Omega} f \cdot v d x+\int_{\Gamma_{N}} g \cdot v d s, \forall v \in H_{\Gamma_{0}}^{1}(\Omega)^{d} \\
\int_{\Omega} A^{-1} \sigma_{\gamma}^{\eta}: \tau d x+\frac{1}{1+\eta} \int_{\Omega} A^{-1} f_{\gamma}\left(1-\frac{\sigma_{c}}{\left|\sigma_{\gamma}^{\eta}\right|_{D}}\right)\left(\sigma_{\gamma}^{\eta}\right)_{D}: \tau d x=\int_{\Omega} e\left(u_{\gamma}^{\eta}\right): \tau d x, \forall \tau \in L^{2}\left(\Omega ; \mathbb{M}_{s}^{d}\right)
\end{array}\right.
$$

\subsubsection{Mathematical analysis}

We give two theorems stating that the solution of (25) exists, is unique and converges to a solution of the Hencky model. For the existence and uniqueness, the proof is the same as the one for proposition 2.8 in [26] with hardening.

Theorem 3.1.1. Under the safe-load condition (14) and $f \in L^{2}(\Omega)^{d}$ and $g \in L^{2}\left(\Gamma_{N}\right)^{d}$, there exists a unique solution $\left(\sigma_{\eta}^{\gamma}, u_{\eta}^{\gamma}\right) \in L^{2}\left(\Omega ; \mathbb{M}_{s}^{d}\right) \times H_{\Gamma_{0}}^{1}(\Omega)^{d}$ to the problem $(25)$.

Theorem 3.1.2. Under the safe-load condition (14) and with $f \in L^{d}(\Omega)^{d}$ and $g \in C^{0}\left(\Gamma_{N}\right)^{d}$, when $\eta \rightarrow 0$ and $\gamma \rightarrow 0$,

- $\sigma_{\gamma}^{\eta}$ converges strongly in $L^{2}\left(\Omega ; \mathbb{M}_{s}^{d}\right)$ to $\sigma$, the stress tensor solution of the Hencky model (13).

- Up to a subsequence, $u_{\gamma}^{\eta}$ converges weakly in $L^{\frac{d}{d-1}}\left(\Omega ; \mathbb{R}^{d}\right)$ and weakly in $B D(\Omega)$ to u the displacement solution of the Hencky model (13).

Proof. The proof is a variation of the one done in [41] chapter 2 and can be found in [42]

\subsection{A second regularization for the von Mises criterion}

\subsubsection{Mathematical analysis}

Another idea to get a compatible model with shape optimization is to focus on the formulation (12), used in numerical applications, and address its non smoothness and ill-posedness. 
Definition 3.1. Let $T$ be the operator defined by

$$
T: u \in H_{\Gamma_{0}}^{1}(\Omega)^{d} \rightarrow T(u) \in\left(H_{\Gamma_{0}}^{1}(\Omega)^{d}\right)^{*}
$$

where $T(u)$ is defined for every $v \in H_{\Gamma_{0}}^{1}(\Omega)^{d}$ as:

$$
\langle T(u), v\rangle=\int_{\Omega} P_{K}^{A^{-1}}(A e(u)): e(v) d x .
$$

Clearly $T$ is monotone but is not coercive. To gain these two properties and the smoothness, we define the following regularized projection:

$$
P_{\gamma}(\tau)=(1+\gamma) \tau-f_{\gamma}\left(1-\frac{\sigma_{c}}{\left|\tau_{D}\right|}\right) \tau_{D}
$$

Definition 3.2. Let $T_{\gamma}$ be the operator defined by

$$
T_{\gamma}: u \in H_{\Gamma_{0}}^{1}(\Omega)^{d} \rightarrow T_{\gamma}(u) \in\left(H_{\Gamma_{0}}^{1}(\Omega)^{d}\right)^{*}
$$

where $T_{\gamma}(u)$ is defined for every $v \in H_{\Gamma_{0}}^{1}(\Omega)^{d}$ as:

$$
\left\langle T_{\gamma}(u), v\right\rangle=\int_{\Omega} P_{\gamma}(A e(u)): e(v) d x .
$$

The function $f_{\gamma}$ in $(27)$ is defined by (17). Then a regularization of (11) is simply

$$
\sigma=P_{\gamma}(A e(u))
$$

\subsubsection{Mathematical analysis}

The study of the operator $T_{\gamma}$ is done in [42], theorems 6.2.9 (strict monotonicity) and 6.2.10 (coercivity), lemmas 6.2.11 (hemicontinuity) and 6.2.12 (boundedness). A simple application of theorem 2.14 in [55] gives:

Theorem 3.2.1. The regularized problem: find $u_{\gamma} \in H_{\Gamma_{0}}^{1}(\Omega)^{d}$ such that,

$$
\int_{\Omega} P_{\gamma}\left(A e\left(u_{\gamma}\right)\right): \epsilon(v) d x=\int_{\Omega} f \cdot v d x+\int_{\Gamma_{N}} g \cdot v d s \forall v \in H_{\Gamma_{0}}^{1}(\Omega)^{d},
$$

admits a unique solution. The associated regularized stress tensor is defined as:

$$
\sigma_{\gamma}=P_{\gamma}\left(A e\left(u_{\gamma}\right)\right) \text {. }
$$

Then it is proved in [42], theorems 6.2.15 and 6.2.16, that the solution of the regularized problem (28) converges to the solution of the Hencky problem, as the regularization parameter goes to zero.

Theorem 3.2.2. Under the safe-load condition (14) and with $f \in L^{d}(\Omega)^{d}$ and $g \in C^{0}\left(\Gamma_{N}\right)^{d}$,

- the solution $\sigma_{\gamma}$ converges, as $\gamma$ goes to 0 , strongly in $L^{2}\left(\Omega ; \mathbb{M}_{s}^{d}\right)$ to $\sigma$, the stress tensor solution of the Hencky model.

- the solution $u_{\gamma}$ converges weakly, up to a subsequence, in $L^{\frac{d}{d-1}}\left(\Omega ; \mathbb{R}^{d}\right)$ and weakly in $B D(\Omega)$ to a displacement u solution to the Hencky model.

\subsection{Conclusion on the two proposed regularizations}

For the von Mises criterion, the two formulations introduced in this section are quite similar. They are tantamount to redefine $\sigma$ by one of these formulae:

1. for the Perzyna penalization

$$
\sigma=A e(u)-\frac{1}{1+\eta} f_{\gamma}\left(1-\frac{\sigma_{c}}{|A e(u)|_{D}}\right)(A e(u))_{D}
$$


2. for the second regularization

$$
\sigma=(1+\gamma) A e(u)-f_{\gamma}\left(1-\frac{\sigma_{c}}{|A e(u)|_{D}}\right)(A e(u))_{D} .
$$

In each case, the problem reduces to a nonlinear variational equation: find $u \in H_{\Gamma_{0}}^{1}(\Omega)^{d}$ such that

$$
\int_{\Omega} \sigma: e(v) d x=\int_{\Omega} f \cdot v d x+\int_{\Gamma_{N}} g \cdot v d s \quad \forall v \in H_{\Gamma_{0}}^{1}(\Omega)^{d} .
$$

\section{Derivation and optimization}

Our goal is to minimize an objective function $J(\Omega)$ depending on $u$, the displacement which solves one of the formulations given in section 3 under constraints also depending on $u$ noted $C(\Omega)$ :

$$
\left\{\begin{array}{l}
\min J(\Omega) \\
\Omega \in \mathcal{U}_{a d} \\
u \text { solution of }(29) \\
C(\Omega) \leq 0
\end{array}\right.
$$

where $\mathcal{U}_{a d}$ is the set of admissible shapes. These shapes should be included into a fixed domain $D$, $\Omega \subset D$, and the Dirichlet boundary $\Gamma_{0} \subset \partial D$ is not allowed to change:

$$
\mathcal{U}_{a d}=\left\{\Omega \subset D \text { bounded and open such that } \Gamma_{0} \subset \partial D \text { is fixed }\right\}
$$

In the following we denote $\Gamma_{m}$ the part of the boundary of $\Omega$ which is allowed to change. Examples of typical functions $J(\Omega)$ and $C(\Omega)$ are given in Remark 4.3.2.

\subsection{Shape derivative}

To minimize (30) we apply a gradient method, which relies on the notion of Hadamard shape derivative for functionals depending on the domain $\Omega$, see for instance [23], [47], [51], [61] or [62]. Starting from a smooth domain $\Omega_{0}$, the variation of the domain takes the form:

$$
\Omega_{\theta}=(I d+\theta)\left(\Omega_{0}\right)
$$

with $\theta \in W^{1, \infty}\left(\mathbb{R}^{d}, \mathbb{R}^{d}\right)$ and $I d$ the identity map. When $\theta$ is sufficiently small, $I d+\theta$ is a diffeomorphism in $\mathbb{R}^{d}$, see [2]. Once the variation of the shape is defined, it is possible to define the notion of Gâteaux derivative for a function $J$ depending on the shape.

Definition 4.1. The shape derivative $J^{\prime}(\Omega)(\theta)$ of $J(\Omega)$ at $\Omega$ in the direction $\theta$ is defined as the derivative at 0 of the application $t \rightarrow J((I d+t \theta)(\Omega))$ which means:

$$
J((I d+t \theta)(\Omega))=J(\Omega)+t J^{\prime}(\Omega)(\theta)+o(t)
$$

where $J^{\prime}(\Omega)$ is a continuous linear form on $W^{1, \infty}\left(\mathbb{R}^{d}, \mathbb{R}^{d}\right)$.

We recall the following classical theorem [2] which will be used in the next section.

Theorem 4.1.1. Let $\Omega$ be a smooth open set, $\phi$ a smooth function defined in $\mathbb{R}^{d}$,

$$
J_{v}(\Omega)=\int_{\Omega} \phi(x) d x \quad \text { and } \quad J_{s}(\Omega)=\int_{\partial \Omega} \phi(x) d s .
$$

These two functions are shape differentiable at $\Omega$ in the direction $\theta \in W^{1, \infty}\left(\mathbb{R}^{d}, \mathbb{R}^{d}\right)$ and

$$
J_{v}^{\prime}(\Omega)(\theta)=\int_{\partial \Omega} \theta \cdot n \phi d s \quad \text { and } \quad J_{s}^{\prime}(\Omega)(\theta)=\int_{\partial \Omega} \theta \cdot n\left(\frac{\partial \phi}{\partial n}+H \phi\right) d s
$$

where $H=\operatorname{div}(n)$ is the mean curvature of $\partial \Omega$. 


\subsection{Differentiability of the regularized formulation}

As far as optimization is concerned, we need to investigate the differentiability of the operator $\tau \rightarrow$ $f_{\gamma}\left(1-\frac{\sigma_{c}}{|\tau|_{D}}\right)$. As $f_{\gamma}$ is a smooth Lipschitz function from $\mathbb{R}$ to $\mathbb{R}$, it is Gateaux differentiable pointwise. It has no chance to be Fréchet differentiable from $L^{2}(\Omega)$ to $L^{2}(\Omega)$.

Lemma 4.2.1. There exists $\delta>0$ such that the solution $u$ of the problem (29) belongs to $W^{1, p}(\Omega)^{d}$ with $p \in[2, \bar{p}]$ and $\bar{p}=2+\delta>2$. Moreover $\tau \rightarrow f_{\gamma}\left(1-\frac{\sigma_{c}}{|\tau|_{D}}\right)$ is Fréchet differentiable from $L^{2+\delta}(\Omega)$ to $L^{2}(\Omega)$.

Proof. The regularity of $u$ is given by theorem 1.1 of [25]. The Fréchet differentiability of $f_{\gamma}$ is done in $[26]$.

This lemma implies that both regularizations are Fréchet differentiable with respect to $u$ from $W^{1, \bar{p}}(\Omega)^{d}$ to $H^{1}(\Omega)^{d}$.

\subsection{Computation of the gradients}

We proceed now to the computation of the gradient of a general criterion:

$$
J(\Omega)=\int_{\Omega} m(u) d x+\int_{\partial \Omega} l(u) d s
$$

where $u$ is solution of $(29), \Gamma_{m}$ is the part of $\partial \Omega$ which is allowed to move during the optimization process, $m$ and $l$ are smooth functions from $\mathbb{R}^{d}$ to $\mathbb{R}$, satisfying the following growth conditions:

$$
|m(u)| \leq C\left(1+|u|^{2}\right), \quad\left|m^{\prime}(u) \cdot h\right| \leq C|u||h|
$$

and

$$
|l(u)| \leq C\left(1+|u|^{2}\right), \quad\left|l^{\prime}(u) \cdot h\right| \leq C|u||h|
$$

for every $h \in L^{2}(\Omega)^{d}$ and $u \in L^{2}(\Omega)^{d}$.

Theorem 4.3.1. Assume that $\Gamma_{m} \cap \Gamma_{0}=\emptyset$, that $f \in H^{1}\left(\mathbb{R}^{d}\right)^{d}$ and $g \in H^{2}\left(\mathbb{R}^{d}\right)^{d}$ and that $u$ is solution of (29). The function $J(\Omega)$, defined by (31), is shape differentiable and its shape derivative is given by

$$
\begin{aligned}
J^{\prime}(\Omega)(\theta) & =\int_{\Gamma_{m}} \theta \cdot n(m(u)-f \cdot p) d s \\
& +\int_{\Gamma_{m}} \theta \cdot n\left(H l(u)+\partial_{n} l(u)\right) \\
& -\int_{\Gamma_{N} \cap \Gamma_{m}} \theta \cdot n\left(H p \cdot g+\partial_{n}(p \cdot g)\right) d s \\
& +\int_{\Gamma_{m}} \theta \cdot n(\sigma: e(p))
\end{aligned}
$$

where $p \in H_{\Gamma_{0}}^{1}(\Omega)^{d}$ is defined as the solution of the following adjoint problem:

$$
\begin{aligned}
& \alpha \int_{\Omega} A e(p): e(\psi) d x-\beta \int_{\Omega} f_{\gamma}\left(1-\frac{\sigma_{c}}{|A e(u)|_{D}}\right)(A e(p))_{D}: e(\psi)_{D} d x \\
& -\beta \int_{\Omega} f_{\gamma}^{\prime}\left(1-\frac{\sigma_{c}}{|A e(u)|_{D}}\right) \frac{\sigma_{c}}{|A e(u)|_{D}^{3}} A e(u)_{D}: A e(\psi)_{D} A e(u)_{D}: e(p)_{D} d x \\
& =-\int_{\Omega} m^{\prime}(u) \cdot \psi d x-\int_{\partial \Omega} l^{\prime}(u) \cdot \psi d s \quad \forall \psi \in H_{\Gamma_{0}}^{1}(\Omega)^{d}
\end{aligned}
$$

with: 
- $\alpha=1$ and $\beta=1 /(1+\eta)$ for the Perzyna regularization,

- $\alpha=1+\eta$ and $\beta=1$ for the second regularization.

Remark 4.3.1. The adjoint problem (33) is well posed for both regularizations using the function (17). This is ensured by the coercivity of the associated bilinear form. To prove it, it suffices to analyze the three possible cases which can occur: $1-\frac{\sigma_{c}}{|A e(u)|_{D}} \in[-\infty,-\gamma], 1-\frac{\sigma_{c}}{|A e(u)|_{D}} \in[-\gamma, \gamma]$ and $1-\frac{\sigma_{c}}{|A e(u)|_{D}} \in$ $[\gamma,+\infty[$.

Proof. The proof is classical and relies on Céa's Lagrangian method [7] or [2]. To make it fully rigorous would require first to prove that the solution $u$ of (29) is Gâteaux differentiable with respect to the shape. This is a well-known result and we simply recall briefly the main arguments. First, the variational formulation (29) is rewritten in the reference configuration $\Omega_{0}$ using a change of variables such that $\Omega=(I d+t \theta)\left(\Omega_{0}\right)$. This leads to a functional equation of the type, $F(u, t)=0$, with $F$ differentiable with respect to $t$. Second, applying the implicit function theorem at $t=0$ yields the desired result (see [23] if necessary). Denoting by $u^{\prime}(\theta)$ the shape derivative of $u$, we now prove the theorem by the Lagrangian method. Let us introduce the Lagrangian $L$, defined for any $v$ and $q$ in $H_{\Gamma_{0}}^{1}\left(\mathbb{R}^{d}\right)^{d}$ (the space of functions defined in $\mathbb{R}^{d}$ which vanishes on $\Gamma_{0}$; recall that $\Gamma_{0}$ is not allowed to move):

$$
\begin{aligned}
L(v, q, \Omega)= & \int_{\Omega} m(v) d x+\int_{\Gamma} l(v) d s+\alpha \int_{\Omega} A e(v): e(q) d x \\
& -\beta \int_{\Omega} f_{\gamma}\left(1-\frac{\sigma_{c}}{|A e(v)|_{D}}\right)(A e(v))_{D}: e(q)_{D} d x \\
& -\int_{\Omega} f \cdot q d x-\int_{\Gamma_{N}} g \cdot q d s
\end{aligned}
$$

with $\alpha$ and $\beta$ depending on the model chosen as stated in Theorem 4.3.1. Since $\Gamma_{0}$ is fixed, there is no need of a Lagrange multiplier for the Dirichlet condition in the Lagrangian: $\Gamma_{0} \subset \partial \Omega$ for every $\Omega \in \mathcal{U}_{a d}$. Moreover the functions $q$ and $v$ are in spaces independent of $\Omega \in \mathcal{U}_{a d}$. Let $(u, p)$ be a stationarity point of $L$. The state equation (29) can be retrieved by differentiating $L$ with respect to $q$ in the direction $\psi \in H_{\Gamma_{0}}^{1}\left(\mathbb{R}^{d}\right)^{d}$ :

$$
\left\langle\partial_{q} L(u, q, \Omega), \psi\right\rangle=0 \quad \forall \psi \in H_{\Gamma_{0}}^{1}\left(\mathbb{R}^{d}\right)^{d}
$$

In the same way the adjoint equation solved by $p$ can be found by derivating $L$ with respect to $v$ in the direction $\psi \in H_{\Gamma_{0}}^{1}\left(\mathbb{R}^{d}\right)^{d}$ :

$$
\begin{aligned}
\left\langle\partial_{u} L, \psi\right\rangle= & \alpha \int_{\Omega} A e(p): e(\psi) d x-\beta \int_{\Omega} f_{\gamma}\left(1-\frac{\sigma_{c}}{|A e(u)|_{D}}\right)(A e(p))_{D}: A e(\psi)_{D} d x+\int_{\Omega} m^{\prime}(u) \cdot \psi d x \\
& +\int_{\Gamma_{m}} l^{\prime}(u) \cdot \psi d s-\beta \int_{\Omega} f_{\gamma}^{\prime}\left(1-\frac{\sigma_{c}}{|A e(u)|_{D}}\right) \frac{\sigma_{c}}{|A e(u)|_{D}^{3}} A e(u)_{D}: A e(\psi)_{D} A e(u)_{D}: e(p)_{D} d x
\end{aligned}
$$

and the adjoint problem can be deduced:

$$
\left\langle\partial_{u} L(u, p, \Omega), \psi\right\rangle=0 \quad \forall \psi \in H_{\Gamma_{0}}^{1}\left(\mathbb{R}^{d}\right)
$$

which gives (33). To find the shape derivative of $J(\Omega)$, we remark that, for any $q \in H_{\Gamma_{0}}^{1}\left(\mathbb{R}^{d}\right)^{d}$,

$$
J(\Omega)=L(u(\Omega), q, \Omega)
$$

and differentiate it with respect to the shape in the direction $\theta$ which gives:

$$
J^{\prime}(\Omega)(\theta)=L^{\prime}\left(\Omega, u_{\Omega}, q\right)(\theta)=\partial_{\Omega} L\left(\Omega, u_{\Omega}, q\right)(\theta)+\left\langle\partial_{u} L\left(\Omega, u_{\Omega}, q\right), u^{\prime}(\theta)\right\rangle .
$$

But as $u^{\prime}(\theta)$ belongs to $H_{\Gamma_{0}}^{1}(\Omega)^{d}$, taking $q=p(\Omega)$ leads to:

$$
\left\langle\partial_{u} L\left(\Omega, u_{\Omega}, p(\Omega)\right), u^{\prime}(\theta)\right\rangle=0 .
$$

Consequently:

$$
J^{\prime}(\Omega, \theta)=\partial_{\Omega} L\left(\Omega, u_{\Omega}, p_{\Omega}\right)(\theta)
$$

and using the formulae of Theorem 4.1.1, we deduce the desired result (32). 
Remark 4.3.2. Our optimization problem (30) involves an objective function $J(\Omega)$ and a constraint $C(\Omega)$. Both functions are of the type of (31) and we now specify the previous formulas for the integrands $m$ and $l$. The objective function $J(\Omega)$ is the volume, corresponding to

$$
\begin{aligned}
& m_{\text {vol }}(u)=1 \\
& l_{\text {vol }}(u)=0 .
\end{aligned}
$$

The constraint $C(\Omega)$ is a criterion on the displacement, restricted to that part of the boundary where the force is applied, namely

$$
\begin{gathered}
m_{\text {Disp }}(u)=0 \\
l_{\text {Disp }}(u)=|u|^{2} \mathbb{1}_{\Gamma_{N}} .
\end{gathered}
$$

\section{Numerical implementation}

\subsection{The level set method}

For numerical purposes our shapes are defined by level set functions, following the framework introduced by Osher and Sethian [49], [48] and [59]. Let $D \subset \mathbb{R}^{d}$ be a bounded domain in which all admissible shapes $\Omega$ are included. The boundary of $\Omega$ is located by virtue of the level set function $\psi$, defined in $D$ by

$$
\begin{cases}\psi(x)=0 & \text { if } x \in \partial \Omega \cap D \\ \psi(x)<0 & \text { if } x \in \Omega \\ \psi(x)>0 & \text { otherwise }\end{cases}
$$

The normal $n$ and the mean curvature $H$ of the shape $\Omega$ are respectively given by $\frac{\nabla \psi}{|\nabla \psi|}$ and $\operatorname{div}\left(\frac{\nabla \psi}{|\nabla \psi|}\right)$. These quantities are computed throughout the whole domain $D$ which naturally defines extensions of their definition on $\partial \Omega$.

\subsection{Optimization algorithm}

This algorithm is similar to that introduced in [3], for a model of linearized elasticity, and the reader is referred to [3] for numerical implementation details. The optimization process produces iteratively a sequence $\left(\Omega_{i}\right)_{i \in \mathbb{N}}$ of shapes, starting from an initial shape $\Omega_{0}$. The level set function is evolved from $\Omega_{i}$ to $\Omega_{i+1}$ by solving the following Hamilton Jacobi transport equation [49] for $t \in\left[0, t_{f}\right]$ :

$$
\frac{\partial \psi}{\partial t}+V|\nabla \psi|=0 \text { in } D
$$

where $\psi(0, x)$ is a level set function for $\Omega_{i}$ and $V(x)$ is the normal velocity of the shape's boundary. This normal velocity $V$ can be identified with $\theta \cdot n$ where $\theta$ is a direction of derivation which makes the shape derivative of section 4 negative. Note that, since shapes derivatives are defined as integrals on the boundary $\partial \Omega$, the normal velocity $V$ must be extended to the whole computational domain $D$. In the mean time, it is also regularized (see e.g. the end of section 3 in [3]). For a constrained optimization problem like (30), we could apply this procedure as such for the Lagrangian, deduce $V$ from the shape derivative of the Lagrangian and update the Lagrange parameters at each optimization iteration. However, for efficiency reasons, we prefer to rely on a Sequential Linear Programming (SLP) type algorithm. More precisely, computing shape derivatives of the objective function and of the constraints (using Theorem 4.3.1), we linearize (30) and, adding a trust region constraint, we solve this linear program using the qld routine in Scilab [58] (a linear quadratic programming solver) to deduce a descent direction which, after extension to $D$, is precisely the normal velocity $V$.

The final time $t_{f}$ for (35) can be interpreted as a descent step. It is initialized as a given value $t_{\max }$ and then decreases until an admissible shape is found (if not the algorithm stops). When an admissible shape is accepted, the time step $t_{f}$ is allowed to grow. We distinguish two phases in the algorithm. First, if the current shape does not fulfil the constraints, we allow the objective to slowly increase as long as the constraints improve. Then, once an admissible shape is reached, we reject every future shape which, 


\begin{tabular}{cccccc}
\hline Case & Volume & Displacement & Constraint & Iter. & Eval. \\
\hline Elastic & 1.35746 & $7.99968 \mathrm{e}-07$ & $8 \mathrm{e}-07$ & 32 & 56 \\
Perzyna & 2.42645 & $7.99909 \mathrm{e}-07$ & $8 \mathrm{e}-07$ & 28 & 51 \\
Sec. Reg. & 2.42656 & $7.99909 \mathrm{e}-07$ & $8 \mathrm{e}-07$ & 28 & 51
\end{tabular}

Table 1: Results for the cantilever

either makes the objective increase or is not admissible. The new shape $\Omega_{i+1}$ is defined by the final level set function $\psi\left(t_{f}, x\right)$.

The Hamilton-Jacobi equation (35) is solved by an explicit second-order upwind scheme on a cartesian grid of $D$ with Neumann boundary conditions. Since the scheme is explicit in time, the time stepping has to satisfy a CFL condition and, in order to regularize the level set which can become too flat or too steep during the successive optimization iterations, periodic reinitializations are performed.

\subsection{Finite element method}

On the same cartesian grid we solve the mechanical system (29) and the adjoint system (33) unsing bilinear quadrangular (Q1) finite elements. Such a choice is done for example in [73], [60] or in [53] where perfect plasticity is solved in the static case and some a posteriori estimates are given. For the two regularized models proposed in this paper, a numerical study is made on their convergence to the Hencky model in [42] (see chapter 4, 4.5.2 for the Perzyna one and chapter 6, 6.2.2 for the other one, using a particular example). Tables with $L^{2}$ errors on $\sigma$ and $u$ are given in [42] as well as tables showing the realization of the constraint $\sigma \in K$. As is well known, using quadrangular elements in the framework of shape optimization can have some drawbacks [65]: the appearance of checkerboard patterns and onenode connections. However, because of the chosen optimization problems, we never found one-node connections in the present study, and recall that there are no checkerboards with the level set method.

To avoid meshing the shapes $\Omega$, we rely on the "ersatz material" approach which fills $D \backslash \Omega$ with a weak material mimicking void but preventing the stiffness matrix from being singular. This technique is commonly used in topology optimization with level sets [3], [70].

Concerning the nonlinear penalized equations, they are usually solved by a damped Newton method, see [12] chapter 6, or a fixed point method. The Newton method has the advantage to be faster but it needs a good choice for the damping. On the other hand, the fixed point method despite its relative slowness is easier to implement. The robustness of the algorithm which solves the direct problem is crucial in the optimization process because the optimization can produce structures for which the finite element matrices are nearly singular. Here we choose to use a fixed point method for the computation of the nonlinear problems, which converges in, at most, 300 iterations with an average of 100 .

\section{$6 \quad$ Numerical examples}

We consider five two-dimensional examples. In all examples the regularization parameters are $\eta=$ $\gamma=10^{-10}$. There are no volume forces but just surface loadings. In every example a small amount of material is forced to remain near the loading and clamped boundaries (these zones cannot be optimized). As a consequence, all shapes exhibit a material layer along the Dirichlet and non-homogeneous Neumann boundaries. For all examples the volume is minimized under an inequality constraint on the displacement (see Remark 4.3.2 for a precise definition). For each example, the value of the constraint can be found in the column "Constraint" of the corresponding table.

\subsection{Cantilever}

For this example we use a grid mesh of 6400 Q1-elements. The design domain $D$ has a length and a height of 2. A constant vertical force equal to 1.1 is applied in the middle of the right side (from $(2,0.9)$ to $(2,1.1)$ ) and the left side is clamped (see Figure 2). The volume is optimized under a displacement constraint. The material parameters are $E=1960, \nu=0.3$ and $\sigma_{c}=0.95$. Results are given in Table 1 and Figures 3 and 4. 


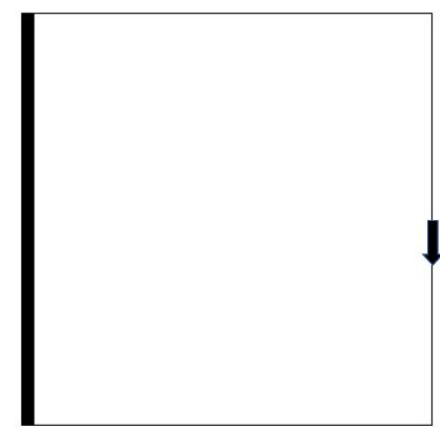

(c) Load case

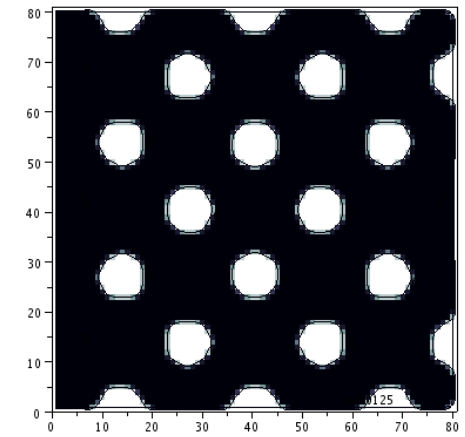

(d) Initialization

Figure 2: Cantilever: problem definition

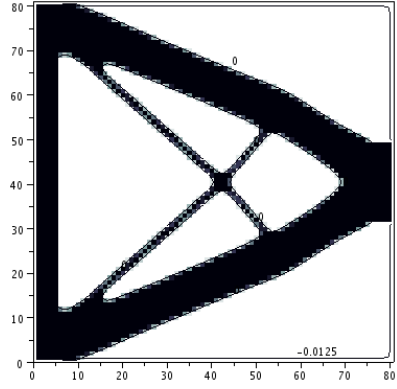

(a) Without plasticity

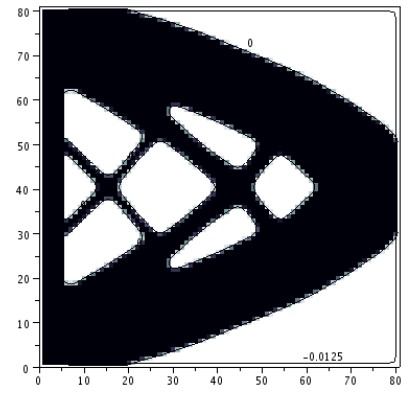

(b) Perzyna penalization

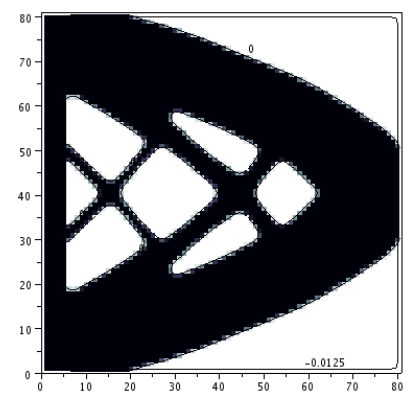

(c) Second regularization

Figure 3: Cantilever: final designs

As can be expected, taking plasticity into account produces heavier structures. Indeed the algorithm tries to avoid the appearance of plastic zones which are less rigid and induce larger displacements. Note that the two different plasticity models give a similar final design and that plasticity zones appear near the boundary conditions.

\subsection{Bridge}

The design domain has a length equal to 4 and a height of 1 . We use a grid of $160 \times 40(6400)$ Q1 elements. The right and left sides are clamped. A constant vertical force equal to 30 is applied on the middle of the upper side from $(1.748,1)$ to $(2.25,1)$ (see Figure 5 ). The volume is optimized under a displacement constraint. The material parameters are $E=1.8 \times 10^{5}, \nu=0$ and $\sigma_{c}=70$, like in [57]. Results are gathered in Table 2 and Figures 6 and 7.

On this example, we note that the algorithm did not take the same path when plasticity is considered. We also remark that plasticity zones appear not only near the loading zone but also at the meeting point of different bars. The fact that, in plastic cases, the volume is lower than in the elastic case could be explained by the different paths taken by the algorithm.

\begin{tabular}{cccccc}
\hline Case & Volume & Displacement & Constraint & Iter. & Eval. \\
\hline Elastic & 1.39414 & $8.99939 \mathrm{e}-07$ & $9 \mathrm{e}-07$ & 47 & 74 \\
Perzyna & 1.38493 & $8.99537 \mathrm{e}-07$ & $9 \mathrm{e}-07$ & 42 & 70 \\
Sec. Reg. & 1.364 & $8.99995 \mathrm{e}-07$ & $9 \mathrm{e}-07$ & 51 & 81
\end{tabular}

Table 2: Results for the bridge 


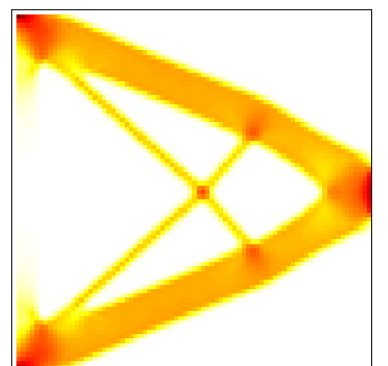

(a) von Mises effective stress for the elasticity case

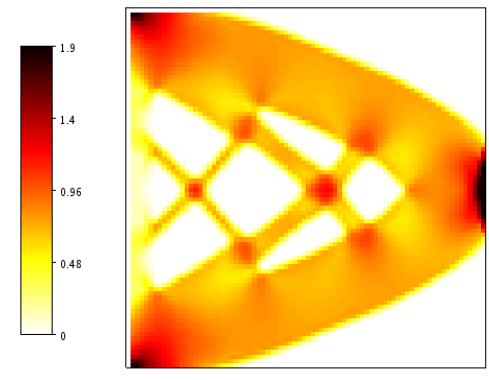

(b) von Mises effective stress for Perzyna penalization
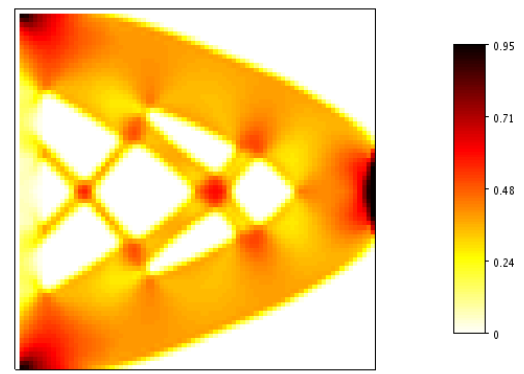

(c) von Mises effective stress for the second regularization

Figure 4: Cantilever: von Mises effective stress plot for the final designs (the color scale differs between elasticity and plasticity).

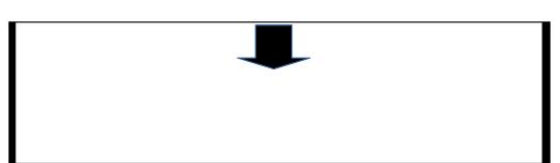

(a) Load case

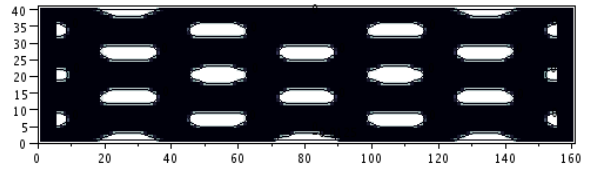

(b) Initialization

Figure 5: Bridge: problem definition

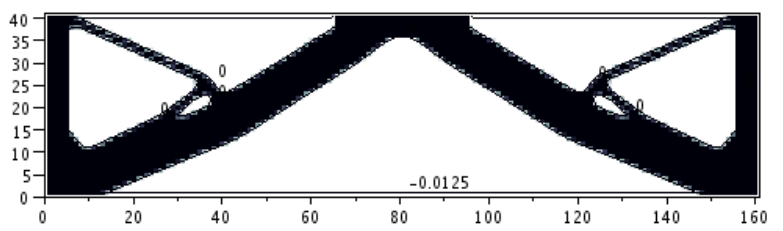

(a) Without plasticity

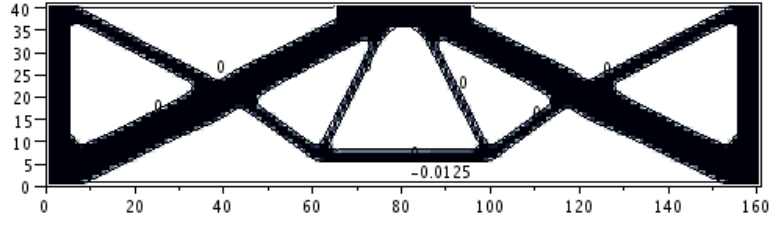

(b) Perzyna penalization

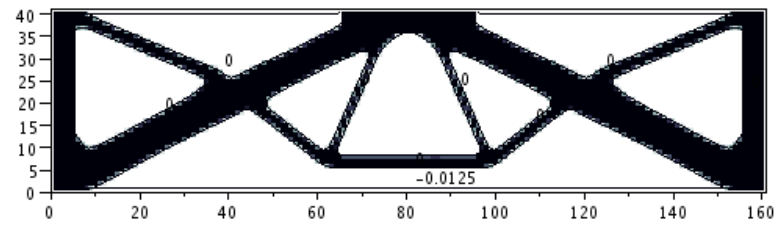

(c) Second regularization

Figure 6: Bridge: final designs

\subsection{Pylon 1}

For this example we use a grid mesh of 6400 Q1-elements. The design domain has a length and a height of 2. The structure is fixed on the bottom right and on the bottom of the left side. A constant vertical 


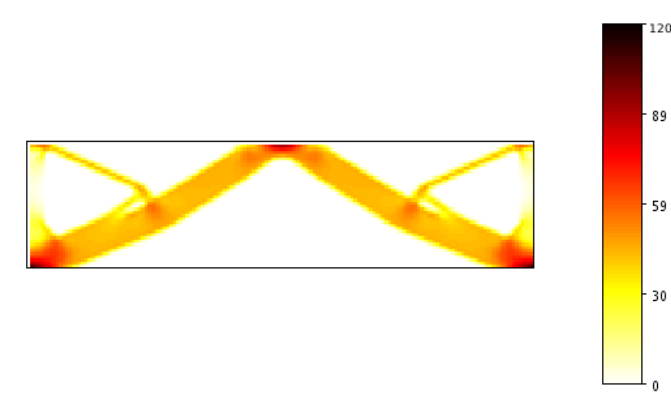

(a) von Mises effective stress for the elasticity case

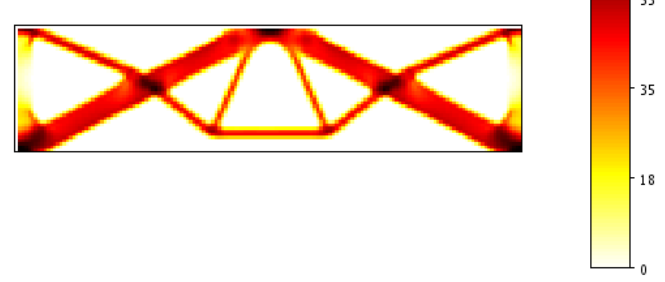

(b) von Mises effective stress for Perzyna penalization

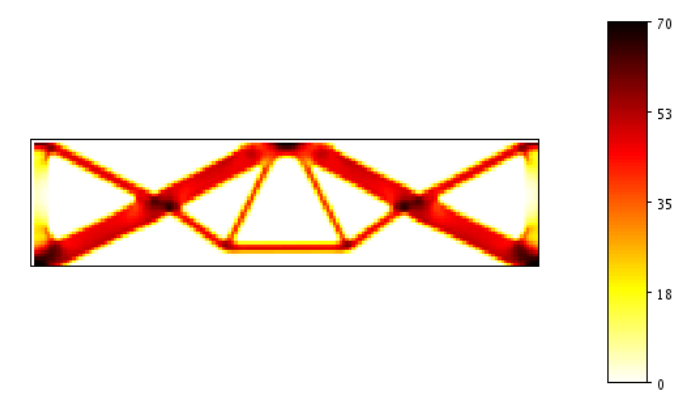

(c) von Mises effective stress for the second regularization

Figure 7: Bridge: von Mises effective stress plot for the final designs (the color scale differs between elasticity and plasticity).

force equal to 2 is applied on the left of the upper side from $(0.1,2)$ to $(0.35,2)$ (see Figure 8 ). The volume is optimized under a displacement constraint. The material parameters are $E=1960, \nu=0.3$ and $\sigma_{c}=2$. Results are shown in Table 3 and Figures 9 and 10 .

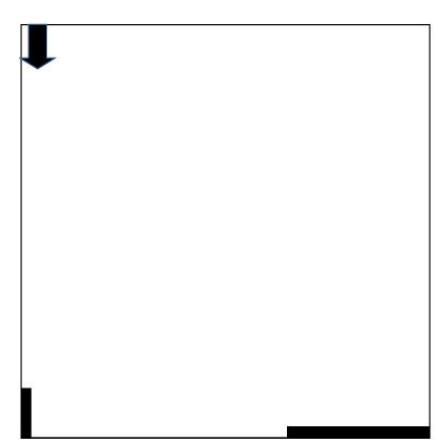

(a) Load case

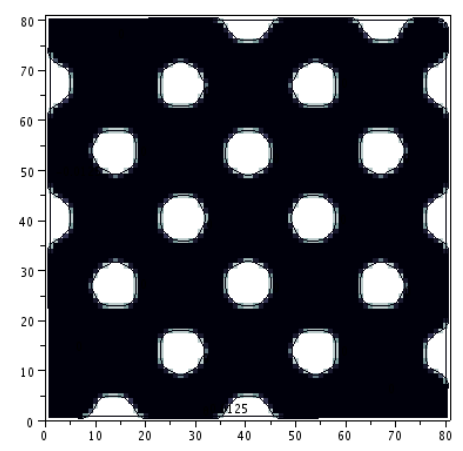

(b) Initialization

Figure 8: Pylon 1: problem definition

On this example, there is a clear difference between the elastic case and the plastic cases. In the elastic case, the connection with the Dirichlet conditions on the bottom right is not needed whereas in the plastic case, it is required by the algorithm. We note also that the final values of the volume are quite the same in every case. 


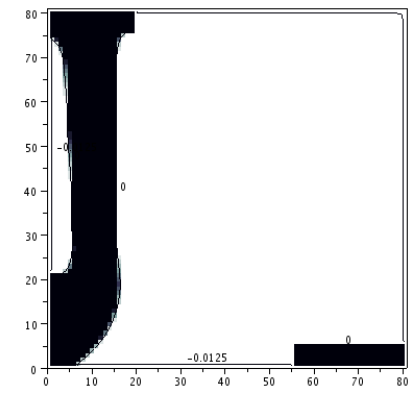

(a) Without plasticity

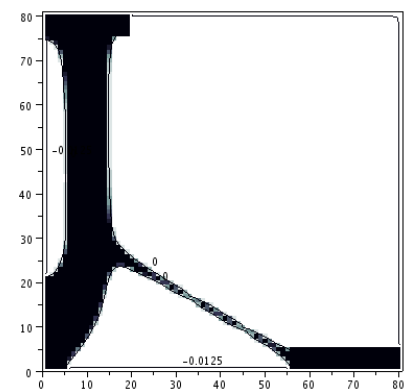

(b) Perzyna penalization

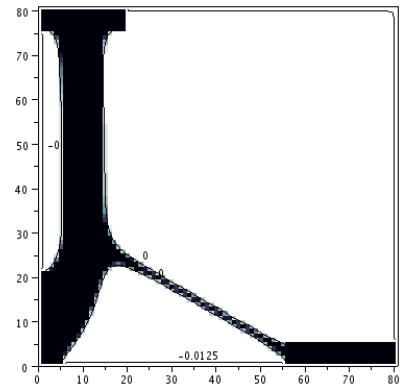

(c) Second regularization

Figure 9: Pylon 1: final designs

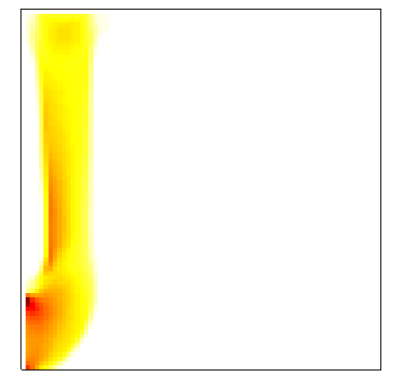

(a) von Mises effective stress for the elasticity case
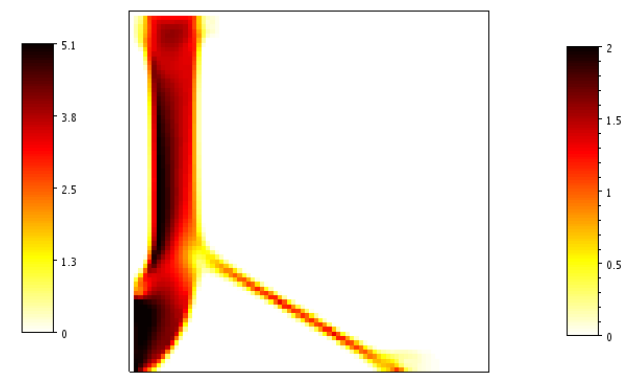

(b) von Mises effective stress for Perzyna penalization
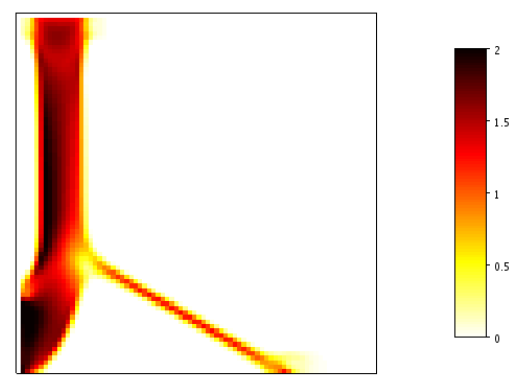

(c) von Mises effective stress for the second regularization

Figure 10: Pylon 1: von Mises effective stress plot for the final designs (the color scale differs between elasticity and plasticity).

\subsection{The $\mathrm{Y}$}

The design domain has a length equal to 2 and a height of 1 . We use a grid of $160 \times 80$ (12800) Q1 elements. The left side is fixed. A constant force equal to 1.3 is applied on the top right side from $(2,0.025)$ to $(2,0.25)$ and on the bottom right side $(2,0.75)$ to $(2,0.975)$ (see Figure 11). The volume is optimized under a displacement constraint. The material parameters are $E=1960, \nu=0.3$ and $\sigma_{c}=1$. Results are collated in Table 4 and Figures 12 and 13.

The elastic is slightly lighter than the plastic ones. We can also remark that the crossed bars in the left middle part of the structure are thinner in the elastic case as they would probably give away if plasticity is taken into account. 


\begin{tabular}{cccccc}
\hline Case & Volume & Displacement & Constraint & Iter. & Eval. \\
\hline Elastic & 0.682463 & $7.99926 \mathrm{e}-07$ & $8 \mathrm{e}-07$ & 602 & 683 \\
Perzyna & 0.682525 & $7.9992 \mathrm{e}-07$ & $8 \mathrm{e}-07$ & 501 & 547 \\
Sec. Reg. & 0.676969 & $7.99838 \mathrm{e}-07$ & $8 \mathrm{e}-07$ & 297 & 349
\end{tabular}

Table 3: Results for the Pylon 1

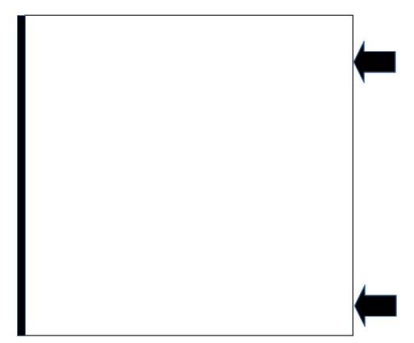

(a) Load case

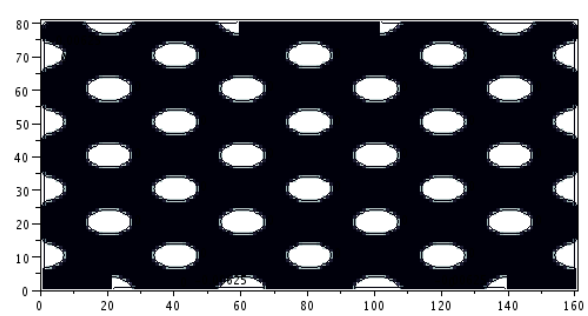

(b) Initialization

Figure 11: The Y

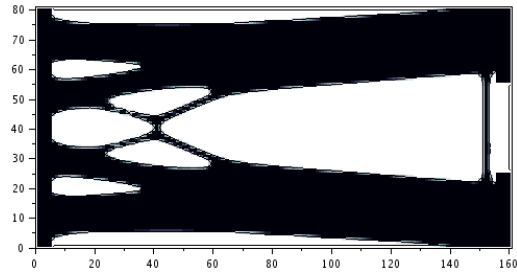

(a) Without plasticity

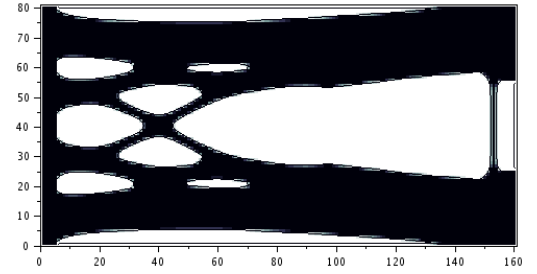

(b) Perzyna penalization

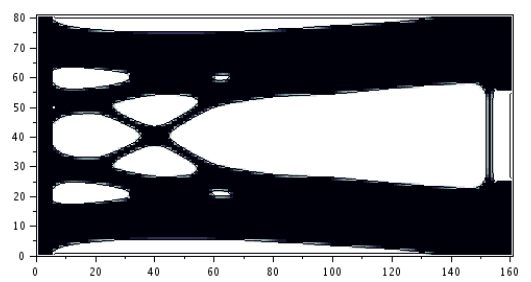

(c) Second regularization

Figure 12: The Y, final designs

\subsection{Pylon 2}

The structure is fixed on the bottom left, right and middle. The design domain has a length equal to 2 and a height of 1 . We use a grid of $160 \times 80(12800)$ Q1 elements. A constant force equal to 40 is applied on the middle of the top from $(0.8,1)$ to $(1.2,1)$. The volume is optimized under a displacement constraint. For the material characteristic we take: $E=3000, \nu=0$ and $\sigma_{c}=70$. Results are presented in table 5 and Figures 15 and 16.

The volume in the elastic case is smaller than in the plastic cases. We point out that the links between the force zone and the two embedded areas on the left and right sides are nearly unnecessary in the elastic case contrary to the plastic cases. Indeed, to hold, the elastic structure mostly needs the Dirichlet zone which is just facing the force no matter smaller it is with respect to the dimension of the zone where the force is applied. In the plastic case, as this Dirichlet zone is four times smaller than the force zone, it leads to the appearing of a plastic area. Consequently, the plastic cases favour to equally hold on each of the three embedded parts. 


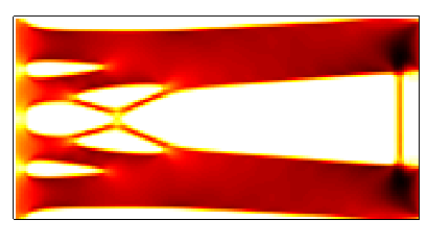

(a) von Mises effective stress for the elasticity case

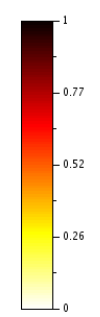

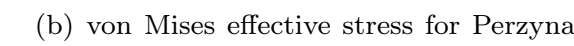
penalization

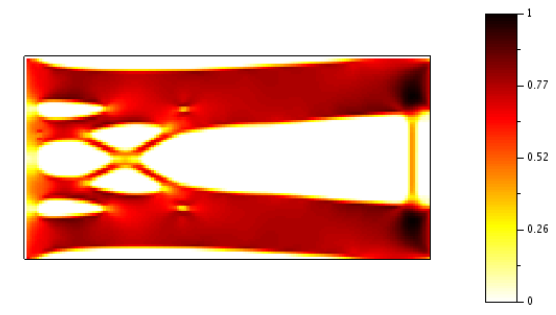

(c) von Mises effective stress for the second regularization

Figure 13: The Y, von Mises effective stress for the final designs (the color scale differs between elasticity and plasticity).

\begin{tabular}{cccccc}
\hline Case & Volume & Displacement & Constraint & Iter. & Eval. \\
\hline Elastic & 1.19588 & $2.99967 \mathrm{e}-07$ & $3 \mathrm{e}-07$ & 77 & 101 \\
Perzyna & 1.21094 & $2.99938 \mathrm{e}-07$ & $3 \mathrm{e}-07$ & 129 & 154 \\
Sec. Reg. & 1.20746 & $2.9913 \mathrm{e}-07$ & $3 \mathrm{e}-07$ & 139 & 163
\end{tabular}

Table 4: Results for the $\mathrm{Y}$

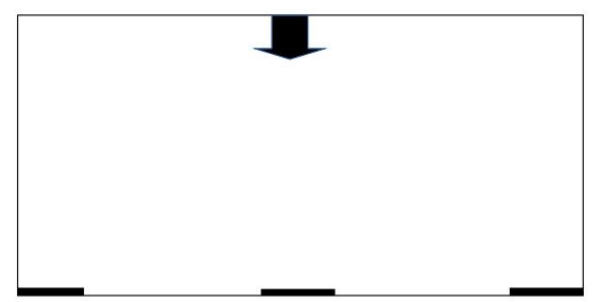

(a) Load case

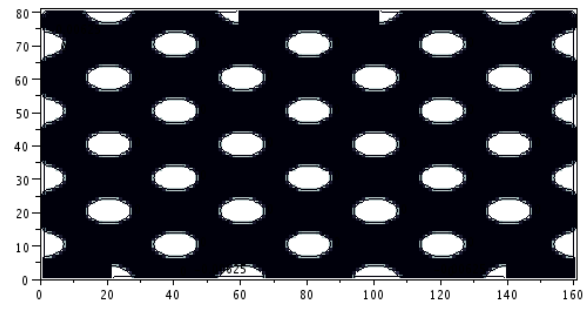

(b) Initialization

Figure 14: Pylon 2

Acknowledgements This work has been supported by the RODIN project (FUI AAP 13). The first author A.Maury has mostly done the work related to this article while being in Laboratoire J.L. Lions (UMR CNRS 7598), University Paris Diderot, Paris, France. The second author G.Allaire is a member of the DEFI project at INRIA Saclay Ile-de-France. 


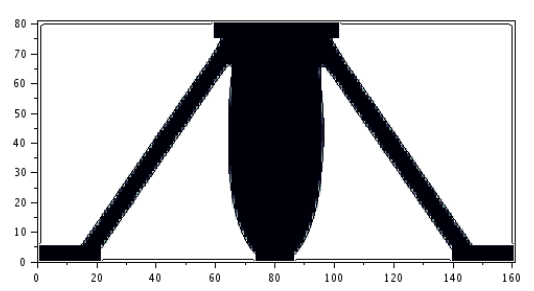

(a) Without plasticity

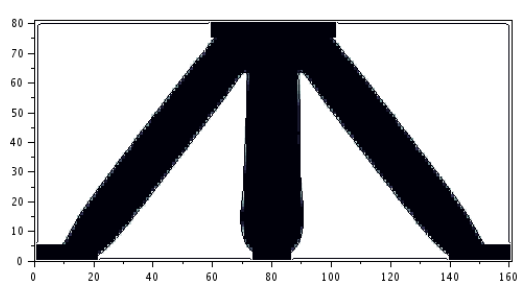

(b) Perzyna penalization

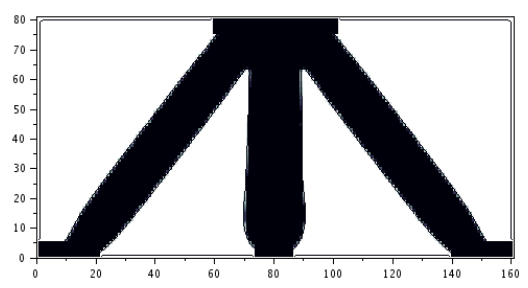

(c) Second regularization

Figure 15: Pylon 2, final designs
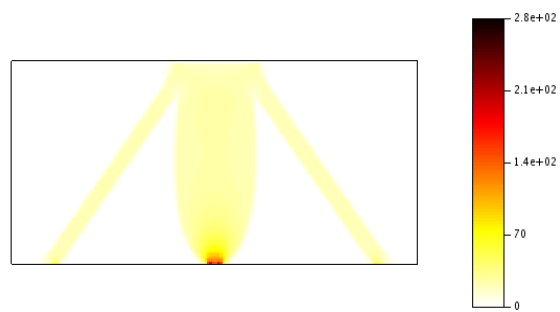

(a) von Mises effective stress for the elasticity case

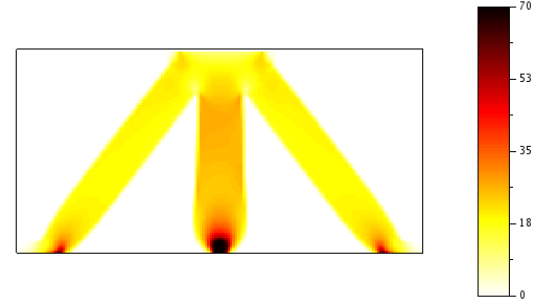

(b) von Mises effective stress for Perzyna penalization

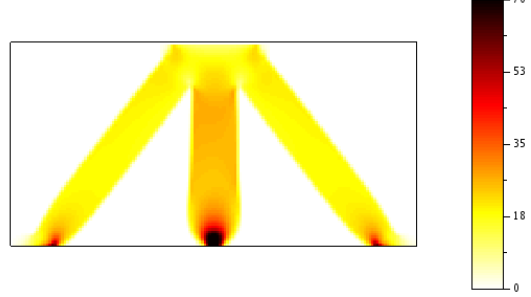

(c) von Mises effective stress for the second regularization

Figure 16: Pylon 2, von Mises effective stress for the final designs (the color scale differs between elasticity and plasticity).

\begin{tabular}{cccccc}
\hline Case & Volume & Displacement & Constraint & Iter. & Eval. \\
\hline Elastic & 0.559843 & $4.99999 \mathrm{e}-07$ & $5 \mathrm{e}-07$ & 39 & 64 \\
Perzyna & 0.690667 & $4.99997 \mathrm{e}-07$ & $5 \mathrm{e}-07$ & 101 & 139 \\
Sec. Reg. & 0.690791 & $4.99999 \mathrm{e}-07$ & $5 \mathrm{e}-07$ & 103 & 143
\end{tabular}

Table 5: Results for the Pylon 2 


\section{References}

[1] B. A. Akbora, R. B. Corotis, and J. H. Ellis. Optimization of structural frames with elastic and plastic constraints. Civil Engineering Systems, 10(2):147-169, 1993.

[2] G. Allaire. Conception optimale de structures, volume 58 of Mathématiques \& Applications (Berlin). Springer-Verlag, Berlin, 2007.

[3] G. Allaire, F. Jouve, and A.-M. Toader. Structural optimization using sensitivity analysis and a level-set method. J. Comput. Phys., 194(1):363-393, 2004.

[4] A. Bensoussan and J. Frehse. Regularity results for nonlinear elliptic systems and applications, volume 151. Springer Science \& Business Media, 2013.

[5] T. Betz and C. Meyer. Second-order sufficient optimality conditions for optimal control of static elastoplasticity with hardening. ESAIM: Control, Optimisation and Calculus of Variations, 21(1):271-300, 2015.

[6] F. Brezzi and M. Fortin. Mixed and hybrid finite element methods, volume 15. Springer Science \& Business Media, 2012.

[7] J. Céa. Conception optimale ou identification de formes, calcul rapide de la dérivée directionnelle de la fonction coût. Modélisation mathématiques et analyse numérique 20-3, pages 371-402, 1986.

[8] G. Dal Maso, A. DeSimone, and M. G. Mora. Quasistatic evolution problems for linearly elasticperfectly plastic materials. Archive for rational mechanics and analysis, 180(2):237-291, 2006.

[9] G. Dal Maso and R. Scala. Quasistatic evolution in perfect plasticity as limit of dynamic processes. Journal of Dynamics and Differential Equations, 26(4):915-954, 2014.

[10] J. C. De los Reyes. Optimization of mixed variational inequalities arising in flow of viscoplastic materials. Computational Optimization and Applications, 52(3):757-784, 2012.

[11] J. C. de los Reyes, R. Herzog, and C. Meyer. Optimal control of static elastoplasticity in primal formulation. SIAM J. Control Optim., 54(6):3016-3039, 2016.

[12] J. J. Dennis and R. Schnabel. Numerical methods for unconstrained optimization and nonlinear equations, volume 16 of Classics in Applied Mathematics. Society for Industrial and Applied Mathematics (SIAM), Philadelphia, PA, 1996. Corrected reprint of the 1983 original.

[13] Z. Dimitrovová. A new methodology to establish upper bounds on open-cell foam homogenized moduli. Structural and Multidisciplinary Optimization, 29(4):257-271, 2005.

[14] G. Duvaut and J. L. Lions. Les inéquations en mécanique et en physique. Dunod, Paris, 1972. Travaux et Recherches Mathématiques, No. 21.

[15] W. Egner, Z. Kordas, and M. Życzkowski. Optimal plastic shape design via the boundary perturbation method. Structural optimization, 8(2-3):145-155, 1994.

[16] M. Fuchs and G. Seregin. Variational methods for problems from plasticity theory and for generalized Newtonian fluids. Springer Science \& Business Media, 2000.

[17] A. Grigusevicius and S. Kalanta. Optimization of elastic-plastic beam structures with hardening using finite element method. Foundations of Civil and Environmental Engineering, (6):31-52, 2005.

[18] P. Grisvard. Elliptic problems in nonsmooth domains, volume 24 of Monographs and Studies in Mathematics. Pitman (Advanced Publishing Program), Boston, MA, 1985.

[19] W. Han and B. D. Reddy. Plasticity: mathematical theory and numerical analysis, volume 9. Springer Science \& Business Media, 2012.

[20] J. Haslinger and R. Mäkinen. Shape optimization of elasto-plastic bodies under plane strains: sensitivity analysis and numerical implementation. Structural optimization, 4(3-4):133-141, 1992. 
[21] J. Haslinger and P. Neittaanmäki. On the existence of optimal shapes in contact problems-perfectly plastic bodies. Computational mechanics, 1(4):293-299, 1986.

[22] J. Haslinger, P. Neittaanmäki, and T. Tiihonen. Shape optimization in contact problems. 1. design of an elastic body. 2. design of an elastic perfectly plastic body. In Analysis and Optimization of Systems, pages 29-39. Springer, 1986.

[23] A. Henrot and M. Pierre. Variation et optimisation de formes, volume 48 of Mathématiques $\&$ Applications (Berlin) [Mathematics \&5 Applications]. Springer, Berlin, 2005. Une analyse géométrique. [A geometric analysis].

[24] R. Herzog and C. Meyer. Optimal control of static plasticity with linear kinematic hardening. ZAMM-Journal of Applied Mathematics and Mechanics/Zeitschrift für Angewandte Mathematik und Mechanik, 91(10):777-794, 2011.

[25] R. Herzog, C. Meyer, and G. Wachsmuth. Integrability of displacement and stresses in linear and nonlinear elasticity with mixed boundary conditions. Journal of Mathematical Analysis and Applications, 382(2):802-813, 2011.

[26] R. Herzog, C. Meyer, and G. Wachsmuth. C-stationarity for optimal control of static plasticity with linear kinematic hardening. SIAM Journal on Control and Optimization, 50(5):3052-3082, 2012.

[27] R. Herzog, C. Meyer, and G. Wachsmuth. B-and strong stationarity for optimal control of static plasticity with hardening. SIAM Journal on Optimization, 23(1):321-352, 2013.

[28] R. Herzog, C. Meyer, and G. Wachsmuth. Optimal control of elastoplastic processes: Analysis, algorithms, numerical analysis and applications. In Trends in PDE Constrained Optimization, pages 27-41. Springer, 2014.

[29] I. Hlaváček. Shape optimization of elastoplastic bodies obeying Hencky's law. Aplikace matematiky, 31(6):486-499, 1986.

[30] I. Hlaváček. Shape optimization of an elasto-perfectly plastic body. Aplikace matematiky, 32(5):381400, 1987.

[31] I. Hlaváček. Shape optimization of elasto-plastic axisymmetric bodies. Applications of Mathematics, 36(6):469-491, 1991.

[32] S. Kaliszky, J. Logo, and T. Havady. Optimal design of elasto-plastic structures under various loading conditions and displacement constraints. Periodica Polytechnica. Civil Engineering, 33(34):107, 1989.

[33] R. Karkauskas. Optimization of elastic-plastic geometrically non-linear lightweight structures under stiffness and stability constraints. Journal of Civil Engineering and Management, 10(2):97-106, 2004 .

[34] J. Kato, H. Hoshiba, S. Takase, K. Terada, and T. Kyoya. Analytical sensitivity in topology optimization for elastoplastic composites. Structural and Multidisciplinary Optimization, 52(3):507$526,2015$.

[35] M. Khanzadi and S. M. Tavakkoli. Optimal plastic design of frames using evolutionary structural optimization. International Journal of Civil Engineering, 9(3), 2011.

[36] A. Khludnev. Optimal control in one-dimensional elastic-plastic models. Journal of applied mechanics and technical physics, 32(5):760-763, 1991.

[37] N. H. Kim, K. K. Choi, and J. Chen. Shape design sensitivity analysis and optimization of elastoplasticity with frictional contact. AIAA Journal, Vol. 38, No. 9, pages 1742-1753, 2000.

[38] N. H. Kim, K. K. Choi, and J. S. Chen. Structural optimization of finite deformation elastoplasticity using continuum-based shape design sensitivity formulation. Computers \& Structures, 79(20):1959$1976,2001$. 
[39] M. Kocvara and J. V. Outrata. Shape optimization of elasto-plastic bodies governed by variational inequalities. Boundary Control and Variation, Lecture Notes in Pure and Applied Mathematics, 163:261-271, 1994.

[40] T. Lee, J. Arora, and V. Kumar. Shape design sensitivity analysis of viscoplastic structures. Computer methods in applied mechanics and engineering, 108(3):237-259, 1993.

[41] D. Löbach. Interior stress regularity for the Prandtl Reuss and Hencky model of perfect plasticity using the Perzyna approximation. Number 386 in Bonner Math. Schriften. Rheinische FriedrichWilhelms-Universität, Mathematisches Institut, 2007.

[42] A. Maury. Shape optimization for contact and plasticity problems thanks to the level set method. Theses, Université Pierre et Marie Curie - Paris VI, Dec. 2016, https://tel.archives-ouvertes.fr/tel01442801/file/2016PA066365.pdf.

[43] K. Maute, S. Schwarz, and E. Ramm. Adaptive topology optimization of elastoplastic structures. Structural Optimization, 15(2):81-91, 1998.

[44] P. Michaleris, D. A. Tortorelli, and C. A. Vidal. Tangent operators and design sensitivity formulations for transient non-linear coupled problems with applications to elastoplasticity. International Journal for Numerical Methods in Engineering, 37(14):2471-2499, 1994.

[45] J. J. Moreau. Evolution problem associated with a moving convex set in a Hilbert space. Journal of Differential Equations, 26(3):347 - 374, 1977.

[46] J. J. Moreau and P. D. Panagiotopoulos. Nonsmooth mechanics and applications, volume 302. Springer, 2014.

[47] F. Murat and J. Simon. Etudes de problèmes d'optimal design. Lecture Notes in Computer Science 41, Springer Verlag, Berlin, pages 54-62, 1976.

[48] S. Osher and R. Fedkiw. Level set methods and dynamic implicit surfaces, volume 153 of Applied Mathematical Sciences. Springer-Verlag, New York, 2003.

[49] S. Osher and J. Sethian. Front propagating with curvature dependent speed: algorithms based on Hamilton-Jacobi formulations. J. Comp. Phys., 78, pages 12-49, 1988.

[50] C. B. Pedersen. Topology optimization of 2d-frame structures with path-dependent response. International Journal for Numerical Methods in Engineering, 57(10):1471-1501, 2003.

[51] O. Pironneau. Optimal shape design for elliptic systems. Springer Series in Computational Physics. Springer-Verlag, New York, 1984.

[52] V. Pištora. Shape optimization of an elasto-plastic body for the model with strain-hardening. Aplikace matematiky, 35(5):373-404, 1990.

[53] R. Rannacher and F.-T. Suttmeier. A posteriori error estimation and mesh adaptation for finite element models in elasto-plasticity. Computer Methods in Applied Mechanics and Engineering, 176(1):333-361, 1999.

[54] E. Rohan and J. Whiteman. Shape optimization of elasto-plastic structures and continua. Computer Methods in Applied Mechanics and Engineering, 187(1):261-288, 2000.

[55] T. Roubíček. Nonlinear partial differential equations with applications, volume 153. Springer Science \& Business Media, 2013.

[56] M. Sauter. Numerical analysis of algorithms for infinitesimal associated and non-associated elastoplasticity. PhD thesis, Karlsruher Inst. für Technologie, Diss., 2010.

[57] S. Schwarz, K. Maute, and E. Ramm. Topology and shape optimization for elastoplastic structural response. Computer Methods in Applied Mechanics and Engineering, 190(15):2135-2155, 2001. 
[58] Scilab Enterprises. Scilab: Le logiciel open source gratuit de calcul numérique. Scilab Enterprises, Orsay, France, 2012.

[59] J. Sethian. Level set methods and fast marching methods, Evolving interfaces in computational geometry, fluid mechanics, computer vision, and materials science, volume 3 of Cambridge Monographs on Applied and Computational Mathematics. Cambridge University Press, Cambridge, second edition, 1999.

[60] J. C. Simo and T. J. Hughes. Computational inelasticity, volume 7. Springer Science \& Business Media, 2006.

[61] J. Simon. Differentiation with respect to the domain in boundary value problems. Num. Funct. Anal. Optimiz, 2, pages 649-687, 1980.

[62] J. Sokolowski and J.-P. Zolesio. Introduction to shape optimization, volume 16 of Springer Series in Computational Mathematics. Springer-Verlag, Berlin, 1992. Shape sensitivity analysis.

[63] P. M. Suquet. Un espace fonctionnel pour les équations de la plasticité. Annales de la Faculté des sciences de Toulouse: Mathématiques, 1(1):77-87, 1979.

[64] P. M. Suquet. Sur les équations de la plasticité: existence et régularité des solutions. J. Mécanique, 20(1):3-39, 1981.

[65] C. Talischi, G. H. Paulino, A. Pereira, and I. F. Menezes. Polygonal finite elements for topology optimization: A unifying paradigm. International journal for numerical methods in engineering, 82(6):671-698, 2010.

[66] L. Tao and D. Zichen. Design optimization for truss structures under elasto-plastic loading condition. Acta Mechanica Solida Sinica, 19(3):264-274, 2006.

[67] R. Temam. Problèmes mathématiques en plasticité, volume 12 of Méthodes Mathématiques de l'Informatique [Mathematical Methods of Information Science]. Gauthier-Villars, Montrouge, 1983.

[68] C. A. Vidal and R. B. Haber. Design sensitivity analysis for rate-independent elastoplasticity. Computer Methods in Applied Mechanics and Engineering, 107(3):393-431, 1993.

[69] G. Wachsmuth. Optimal control of quasi-static plasticity with linear kinematic hardening, part i: Existence and discretization in time. SIAM Journal on Control and Optimization, 50(5):2836-2861, 2012.

[70] M. Y. Wang, X. Wang, and D. Guo. A level set method for structural topology optimization. Computer methods in applied mechanics and engineering, 192(1):227-246, 2003.

[71] K. Washizu. Variational Methods in Elasticity and Plasticity. 01. Elsevier Science \& Technology, 1974.

[72] C. Wieners. Orthogonal projections onto convex sets and the application to problems in plasticity. Citeseer, 1999.

[73] C. Wieners. Nonlinear solution methods for infinitesimal perfect plasticity. ZAMM-Journal of Applied Mathematics and Mechanics/Zeitschrift für Angewandte Mathematik und Mechanik, 87(89):643-660, 2007.

[74] K. Yuge and N. Kikuchi. Optimization of a frame structure subjected to a plastic deformation. Structural optimization, 10(3-4):197-208, 1995.

[75] E. H. Zarantonello. Projections on convex sets in Hilbert space and spectral theory. University of Wisconsin, 1971. 